\title{
Oncology and psychiatry intersect
}

\section{Depression, SSRIs, cancer and drug interactions}

\author{
Kara Marie Ruicci, Dominic Wang
}

\section{ABSTRACT}

Major depressive disorder (MDD) is a highly prevalent and critically disabling medical condition. Numerous factors have been associated with the onset of MDD, including both environmental and heritable components. Depression has been found to be common among cancer patients, who often face uncertain treatment courses. In the present case, a 51-yearold woman presents to her family physician with an ongoing episode of severe depression, characterized by low energy, insomnia and speech latency. In addition to a past medical history significant for MDD, this woman has a 1-year history of estrogen-receptor positive invasive ductal carcinoma (IDC). She previously underwent a unilateral mastectomy and reconstruction is pending. As she began adjuvant oral tamoxifen therapy, her ongoing selective serotonin reuptake inhibitor (SSRI) was changed from fluoxetine to escitalopram, owing to the propensity for drug interactions between certain SSRIs (including fluoxetine) and the cytochrome P450 (CYP) 2D6 enzyme that is responsible for metabolizing tamoxifen into its active metabolite. Over the course of her recent escitalopram treatment, this woman's depressive symptoms have returned. Following assessment, including the Patient Health Questionnaire (PHQ)-9, this woman is referred to adult outpatient psychiatry for management. This case illustrates some of the major features of MDD, and uniquely focuses on the balance required in managing a patient's depression and maintaining their cancer treatment program. A discussion of factors contributing to the onset of depression in cancer patients is included, with focus on the role of reconstructive surgeries for breast cancer patients.

\section{CASE PRESENTATION}

The following case has been created by the authors, and is not based on a real-life patient encounter.

Ms. Patmore is a 51-year-old premenopausal female who presents to her family physician's office with an ongoing episode of severe depression. She is accompanied by her daughter, who reports her mother's inability to sleep, a lack of appetite, low energy and speech latency. ${ }^{1}$ Past medical history is significant for major depressive disorder (MDD) diagnosed five years ago and managed effectively in the past three years with the selective serotonin reuptake inhibitor (SSRI) fluoxetine (20 mg per day). Ms. Patmore's daughter reports their family's recent concerns that Ms. Patmore is becoming increasingly depressed, with similar characteristics as her initial depressive episodes five years previously.
Ms. Patmore has a recent (1 year) history of estrogen-receptor positive invasive ductal carcinoma (IDC; T1N1 disease) that was identified on routine mammogram. She underwent a unilateral mastectomy 10 months ago and surgical reconstruction is pending. Post-operatively, Ms. Patmore began adjuvant therapy with oral tamoxifen (20 mg per day) as her cancer was found to be estrogen receptor (ER) positive. ${ }^{2}$ Her daughter reports that at that time, fluoxetine was replaced with the SSRI escitalopram $(20 \mathrm{mg}$ per day), owing to the propensity for drug interactions between certain SSRIs (including fluoxetine) and the cytochrome P450 (CYP) 2D6 enzyme, which metabolizes tamoxifen to its active metabolite, endoxifen. ${ }^{3}$ Over the past three months, Ms. Patmore's depressive symptoms have returned and worsened.

\section{INITIAL INVESTIGATIONS}

Ms. Patmore is presenting with characteristic signs of MDD (lack of appetite, depressed mood, insomnia, fatigue). ${ }^{1}$ Her medical history suggests that her recent change in SSRI may have affected her, as well as the recent physical and psychological stresses related to her cancer diagnosis and mastectomy. On arrival, her vitals are within normal limits. An electrocardiogram (ECG) and bloodwork (including $\mathrm{CBC}$, a comprehensive metabolic panel and thyroid stimulating hormone (TSH)), are ordered to investigate a mood disorder secondary to an underlying medical cause, such as hypothyroidism. ${ }^{4}$ Ms. Patmore's daughter denies any history of mania or bipolar disorder, as well as use of illicit drugs, alcohol, or cannabis consumption. ${ }^{4,5}$

The Patient Health Questionnaire (PHQ)-2 is administered and found to be positive. The PHQ-9 is subsequently administered and is positive for severe depression (total score $>20) .{ }^{6}$ Owing to her history of MDD and use of SSRIs, Ms. Patmore is referred to adult outpatient general psychiatry for further assessment and treatment planning.

\section{MDD \& DEPRESSION IN CANCER}

MDD is a highly prevalent and critically disabling medical condition worldwide. ${ }^{7}$ MDD is characterized by a depressed mood, a lack of interests, impaired concentration, fatigue, suicidal ideation and often disturbed sleep and/or eating patterns. ${ }^{1}$ MDD affects twice as many women as men, causing considerable impairment. ${ }^{8}$ The disorder not only causes a reduction in health, similar to other chronic disease (e.g. arthritis, asthma, diabetes), but also substantially worsens health when it is co-morbid with these diseases. ${ }^{9}$ As such, health care providers should not overlook the presence of depression in patients who have chronic physical disorders. ${ }^{910}$ Numerous factors have been associated with the 
development of MDD, including both environmental and heritable components. ${ }^{8}$ Presently, MDD is managed using pharmacological treatments and/or psychotherapy, both of which have demonstrated efficacy. ${ }^{11}$ Second generation antidepressants such as SSRIs and selective serotonin norepinephrine reuptake inhibitors (SNRIs) are the most commonly used pharmacological agents. ${ }^{12}$ Cognitive behavioral therapy (CBT) is the foremost form of psychotherapy and focuses on modifying maladaptive thinking patterns through structured, goal-oriented sessions. ${ }^{13}$

Depression is common among cancer patients, with prevalence rates as much as four times higher than in the general population. ${ }^{14}$ It has been suggested that co-morbid depression may be associated with worse prognoses, as well as increased mortality rates. ${ }^{15}$ Depressive symptoms typically develop during the first year after cancer diagnosis and are in large part thought to extend from the psychological distress faced by patients. ${ }^{16}$ Patients facing longlasting, demanding and uncertain treatments experience shock and stress, providing an opportunity for psychological instability. ${ }^{17}$ Physical changes experienced by cancer patients, including chemotherapy-induced alopecia, have also been significantly associated with depression. ${ }^{18}$ The burden of emotional pain and distress patients and their families experience dealing with cancer has led to the creation of a new cancer care field called psychosocial oncology, or psycho-oncology. ${ }^{19}$ Psychosocial oncology programs focus on interventions to improve patients' experiences, by providing supports that address the social, psychological, emotional, spiritual and functional aspects of patients' lives.

For patients facing breast cancer specifically, the profound mutilating effects of surgery - even when completed with curative intent - can dramatically influence a patient's body image and psychological well-being. ${ }^{20}$ Partial and complete mastectomy of diseased and contralateral breasts has become increasingly frequent, in part related to the increasing use of breast MRI (magnetic resonance imaging) for screening. ${ }^{21}$ The co-occurrence between mastectomy and depression in breast cancer survivors has been described. ${ }^{22}$ Breast reconstruction is therefore increasingly a solution for breast cancer patients who undergo ablative surgery in order to help restore patients' sense of self, as is access to psychosocial oncology services.

\section{CYP2D6 INTERACTIONS WITH TAMOXIFEN}

Owing to the prevalence of depression and MDD in cancer patients, efforts are increasingly being made to manage patients' depressive symptoms as they undergo their cancer treatment. Many considerations are taken into account with regard to the pharmacological management of depression for a given patient. In breast cancer, where many patients receive post-operative hormone therapy (estrogen receptor blockers or aromatase inhibitors) to reduce the risk of recurrence, consideration is required regarding which antidepressants are simultaneously prescribed. ${ }^{4}$ An example is in the case of the anti-estrogen drug tamoxifen. Tamoxifen is metabolized by CYP2D6, which converts tamoxifen into its active metabolite endoxifen. ${ }^{3}$ A subset of anti-depressant medications function as inhibitors of CYP2D6, including the SSRIs paroxetine and fluoxetine, as well as bupropion, a norepinephrine-dopamine reuptake inhibitor. ${ }^{4}$ As such, the use of antidepressants targeting CYP2D6 should be reviewed in cancer patients receiving hormonal therapy with tamoxifen, given alternative antidepressants exist. The SSRIs citalopram and escitalopram, the SNRIs desvenlafaxine and venlafaxine, and the noradrenergic and specific serotonergic antidepressants (NaSSAs) such as mirtazapine are weak/nonCYP2D6 inhibitors and therefore safer treatment options unlikely to alter the efficacy of tamoxifen when co-prescribed. ${ }^{23-25}$ Of note, alternatives to tamoxifen (such as the aromatase inhibitors (AIs) anastrozole and exemestane) may be prescribed to postmenopausal patients, as these agents are not affected by anti-depressant medications. $^{26}$ However, AIs are not generally prescribed to premenopausal patients whose ovaries still produce estrogen. Instead, premenopausal patients with estrogen receptor expression are likely to receive tamoxifen, either alone or in combination with either chemical ovarian suppression (via luteinizing hormonereleasing hormone (LHRH) agonists, such as goserelin) or ablation. ${ }^{26}$

\section{CASE CONTINUATION}

Ms. Patmore is evaluated by adult outpatient general psychiatry for MDD according to the Diagnostic and Statistical Manual of Mental Disorders, Fifth Edition (DSM-5). A diagnosis of MDD is confirmed. Mirtazapine (15 mg per day) is prescribed to replace Ms. Patmore's current antidepressant, escitalopram. Although daily fluoxetine previously worked well for Ms. Patmore, it is a strong CYP2D6 inhibitor, making it a non-ideal choice alongside daily tamoxifen which she will continue to take for up to 5 years. ${ }^{27}$ Mirtazapine is known to have a rapid onset and good tolerability, with efficacy apparent as early as two weeks after treatment initiation. ${ }^{25}$ Additionally, mirtazapine promotes appetite and is less likely to cause nausea or vomiting compared to other antidepressants (due to blockade of 5-HT3 receptors). ${ }^{25,28}$ The possibility of adding psychotherapy to Ms. Patmore's treatment plan is also discussed. Studies examining combined pharmacological treatment and psychotherapy suggest that the combination may be more effective than treating MDD with either modality alone. ${ }^{29}$ Regular outpatient follow-up appointments are scheduled to monitor the efficacy of mirtazapine and explore additional management possibilities, including psychotherapy.

\section{CONCLUSIONS}

At follow up over the next several weeks, Ms. Patmore's MDD is found to be improving with mirtazapine. The initial signs at presentation (insomnia, lack of appetite, fatigue and speech latency) all show gradual improvement with daily mirtazapine. Ms. Patmore returns for follow-up appointments through outpatient psychiatry. She also continues to be monitored by her family physician and medical oncologist regarding her tamoxifen therapy and any signs of cancer recurrence. Breast reconstruction is scheduled in 6 months' time.

In summary, this case explores a 51 year-old premenopausal patient with poorly controlled MDD following a change in antidepressant medication intended to avoid a potential drug interaction with tamoxifen via the enzyme CYP2D6. 


\section{REFERENCES}

1. Uher R, Payne JL, Pavlova B, et al. Major depressive disorder in DSM-5: implications for clinical practice and research of changes from DSM-IV. Depress Anxiety. 2014 Jun;31(6):459-71. https://doi. org $/ 10.1002 /$ da. 22217

2. Allred DC, Anderson SJ, Paik S, et al. Adjuvant tamoxifen reduces subsequent breast cancer in women with estrogen receptor-positive ductal carcinoma in situ: a study based on NSABP protocol B-24. J Clin Oncol. 2012 Apr;30(12):1268-73. https://doi.org/10.1200/ JCO.2010.34.0141

3. Cronin-Fenton DP, Damkier P, Lash TL. Metabolism and transport of tamoxifen in relation to its effectiveness: new perspectives on an ongoing controversy. Future Oncol. 2014;10(1):107-22. https://doi. org/10.2217/fon.13.168

4. McMichael KS, Adams K, Breden Crouse EL. Tamoxifen and depression: drug interactions in breast cancer. Consult Pharm. 2013 Sept;28(9):584-91. https://doi.org/10.4140/TCP.n.2013.584

5. De Berardis D, Brucchi M, Serroni N, et al. Successful use of agomelatine in the treatment of major depression in a woman taking tamoxifen: a case report. Clin Neuropharmacol. 2014 JanFeb;37(1):31-3.

6. Maurer DM. Screening for depression. Am Fam Physician. 2012 Jan;85(2):139-44.

7. Kessler RC Berglund P, Demler O, et al. The epidemiology of major depressive disorder: results from the National Comorbidity Survey Replication (NCS-R). JAMA. 2003 Jun;289(23):3095-105. https://doi. org/10.1001/jama.289.23.3095

8. Otte C, Gold SM, Penninx BW, et al. Major depressive disorder. Nat Rev Dis Primers. 2016 Sept;2:16065. https://doi.org/10.1038/ nrdp. 2016.65

9. Moussavi S, Chatterji S, Verdes E, et al. Depression, chronic diseases, and decrements in health: results from the World Health Surveys. Lancet. 2007 Sept;370(9590):851-8. https://doi.org/10.1016/s01406736(07)61415-9

10. Kupfer DJ, Frank E, Phillips ML. Major depressive disorder: new clinical, neurobiological, and treatment perspectives. Lancet. 2012 Mar;379(9820):1045-55. https://doi.org/10.1016/s0140-6736(11)60602$\underline{8}$

11. Cuijpers P, Sijbrandij M, Koole SL, et al. The efficacy of psychotherapy and pharmacotherapy in treating depressive and anxiety disorders: a meta-analysis of direct comparisons. World Psychiatry. 2013 Jun;12(2):137-48.

12. Gartlehner G, Wagner G, Matyas N, et al. Pharmacological and nonpharmacological treatments for major depressive disorder: review of systematic reviews. BMJ Open. 2017;7(6):e014912. https://doi. org/10.1136/bmjopen-2016-014912

13. Driessen E, Hollon SD. Cognitive behavioral therapy for mood disorders: efficacy, moderators and mediators. Psychiatr Clin North Am. 2010 Sept;33(3):537-55. https://doi.org/10.1016/j.psc.2010.04.005

14. Lutgendorf SK, Andersen BL. Biobehavioral approaches to cancer progression and survival: mechanisms and interventions. Am Psychol. 2015 Feb-Mar;70(2):186-97. https://doi.org/10.1037/a0035730

15. Satin JR, Linden W, Phillips MJ. Depression as a predictor of disease progression and mortality in cancer patients: a meta-analysis. Cancer. 2009 Nov;115(22):5349-61. https://doi.org/10.1002/cncr.24561
16. Bortolato B, Hyphantis TN, Valpione S, et al. Depression in cancer: the many biobehavioral pathways driving tumor progression. Cancer Treat Rev. 2017 Jan;52:58-70. https://doi.org/10.1016/j.ctrv.2016.11.004

17. de Medeiros MC, Veiga DF, Sabino Neto M, et al. Depression and conservative surgery for breast cancer. Clinics. 2010;65(12):1291-4. https://doi.org/10.1590/s1807-59322010001200011

18. Choi EK, Kim IR, Chang O, et al. Impact of chemotherapyinduced alopecia distress on body image, psychosocial well-being, and depression in breast cancer patients. Psychooncology. 2014 Oct;23(10):1103-10. https://doi.org/10.1002/pon.3531

19. Bultz BD. Cancer care and the role of psychosocial oncology: where are we and where are we going? Asia Pac J Oncol Nurs. 2016;3(2):118-20.

20. de Raaff CA, Derks EA, Torensma B, et al. Breast reconstruction after mastectomy: does it decrease depression at the long-term? Gland Surg. 2016 Aug;5(4):377-84. https://doi.org/10.21037/gs.2016.05.02

21. Lobbes MB, Vriens IJ, van Bommel AC, et al. Breast MRI increases the number of mastectomies for ductal cancers, but decreases them for lobular cancers. Breast Cancer Res Treat. 2017 Jan;162(2):353-64. https://doi.org/10.1007/s10549-017-4117-8

22. Kim MS, Kim SY, Kim JH, et al. Depression in breast cancer patients who have undergone mastectomy: a national cohort study. PLoS One. 2017 Apr;12(4):e0175395. https://doi.org/10.1371/journal.pone.0175395

23. Aguiar CC, Aguiar JV, Rocha MG. Treatment of depression in patients under breast cancer therapy: antidepressant-tamoxifen drug interactions. J Drug Metab Toxicol. 2017 May;8(2):1000228. https:// doi.org/10.4172/2157-7609.1000228

24. Stimmel GL, Dopheide JA, Stahl SM. Mirtazapine: an antidepressant with noradrenergic and specific serotonergic effects. Pharmacotherapy. 1997 Jan-Feb;17(1):10-21.

25. Watanabe N, Omori IM, Nakagawa A, et al. Mirtazapine versus other antidepressive agents for depression. Cochrane Database Syst Rev. 2011 Dec;(12):CD006528. https://doi.org/10.1002/14651858.CD006528.pub2

26. Kadakia KC, Henry NL. Adjuvant endocrine therapy in premenopausal women with breast cancer. Clin Adv Hematol Oncol. 2015 Oct;13(10):663-72.

27. Gelenberg AJ, Freeman MP, Markowitz JC, et al. Practice guideline for the treatment of patients with major depressive disorder. 3rd ed. Arlington (VA): American Psychiatric Association; 2010. 152 p.

28. Alam A, Voronovich Z, Carley JA. A review of therapeutic uses of mirtazapine in psychiatric and medical conditions. Prim Care Companion CNS Disord. 2013;15:5. https://doi.org/10.4088/ PCC.13r01525

29. Cuijpers P, Sijbrandij M, Koole SL, et al. Adding psychotherapy to antidepressant medication in depression and anxiety disorders: a meta-analysis. World Psychiatry. 2014 Feb;13:56-67. https://doi. org/10.1002/wps.20089 